\title{
A Metal-Free Oxygenated Covalent Triazine 2-D Photocatalyst Works Effectively from the Ultraviolet to Near-Infrared Spectrum for Water Oxidation Apart from Water Reduction
}

\author{
Dan Kong, Xiaoyu Han, Stephen A. Shevlin, Christopher Windle, Jamie H. Warner, Zheng-Xiao Guo,* \\ and Junwang Tang*
}

Cite This: https://dx.doi.org/10.1021/acsaem.0c01153

Read Online

ACCESS |

|lll Metrics \& More

Article Recommendations

Supporting Information

ABSTRACT: Solar-driven water splitting is highly desirable for hydrogen fuel production, particularly if water oxidation is effectively sustained in a complete cycle and/or by means of stable and efficient photocatalysts of main group elements, for example, carbon and nitrogen. Despite extensive success on $\mathrm{H}_{2}$ production on polymer photocatalysts, polymers have met with very limited success for the rate-determining step of the water splitting-water oxidation reaction due to the extremely slow "fourhole" chemistry. Here, the synthesized metal-free oxygenated covalent triazine (OCT) is remarkably active for oxygen production in a wide operation window from UV to visible and even to NIR (up to $800 \mathrm{~nm}$ ), neatly matching the solar spectrum

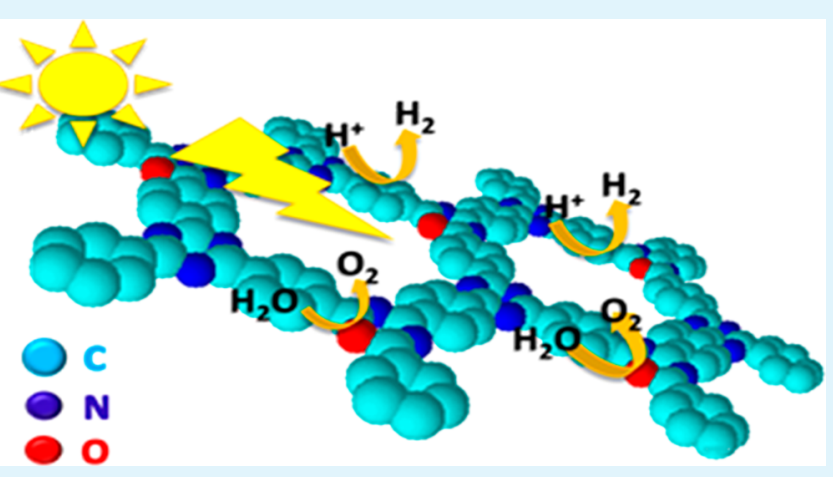
with an unprecedented external quantum efficiency (even 1\% at $600 \mathrm{~nm}$ ) apart from excellent activity for $\mathrm{H}_{2}$ production under full arc irradiation, a big step moving toward full solar spectrum water splitting. Experimental results and DFT calculations show that the oxygen incorporation not only narrows the band gap but also causes appropriate band-edge shifts. In the end, a controlled small amount of oxygen in the ionothermal reaction is found to be a promising and facile way of achieving such oxygen incorporation. This discovery is a significant step toward both scientific understanding and practical development of metal-free photocatalysts for cost-effective water oxidation and hydrogen generation over a large spectral window.

KEYWORDS: oxygen doping, covalent triazine frameworks, photocatalytic oxygen production, IR radiation, wide operation window, hydrogen generation

\section{INTRODUCTION}

Photocatalytic hydrogen production directly from water allows the conversion of solar energy into renewable and clean chemical energy and thus provides a potential solution to energy security and $\mathrm{CO}_{2}$ emission reduction. ${ }^{1}$ There have been numerous studies on semiconductor photocatalysts for either hydrogen or oxygen evolution half-reaction over the past decades, ${ }^{2-8}$ which is the key for both photoelectrochemical water splitting and $\mathrm{Z}$-scheme water splitting that can simultaneously produce spatially separated $\mathrm{H}_{2}$ and $\mathrm{O}_{2}$ gas. However, the majority of these photocatalysts are inorganic materials. ${ }^{9}$ It is only in the past decade that metal-free photocatalysts have been developed, including graphite oxide, ${ }^{10} \mathrm{~g}_{-} \mathrm{C}_{3} \mathrm{~N}_{4}$, ${ }^{11,12}$ and conjugated polymers. ${ }^{13,14}$ The latter ones usually show excellent photocatalytic performance for hydrogen production but are rather poor or moderate for water oxidation (or oxygen generation) because of a too large band gap and/or inappropriate band edges, especially not efficient at the strongest solar insolation range from 400 to $700 \mathrm{~nm} .^{10,15}$
The oxygen evolution process involves both $4 \mathrm{e}^{-} / 4 \mathrm{H}^{+}$ electrochemistry and $\mathrm{O}=\mathrm{O}$ bond formation. As such, it is much more challenging than proton reduction to hydrogen and is the rate-determining step in water splitting. ${ }^{13,16}$ Currently, a few metal oxides (e.g., $\mathrm{Ag}_{3} \mathrm{PO}_{4}$ and $\mathrm{BiVO}_{4}$ ) are reported to oxidize water under visible light, albeit with a relatively narrow operation window (from UV up to $560 \mathrm{~nm}$ ), which does not match well with the solar spectrum and thus limits their efficiency. ${ }^{17-20}$ Unmodified organic photocatalysts usually show negligible activity for water oxidation, typically due to high-lying valence bands that are too close to the water oxidation potential, for example, $\mathrm{g}-\mathrm{C}_{3} \mathrm{~N}_{4} \cdot{ }^{11,12}$ Band gap

Received: June 16, 2020

Accepted: August 24, 2020

Published: August 24, 2020 
engineering to modify the optical properties of organic photocatalysts has been experimentally and theoretically proposed, for example, via addition of comonomers, ${ }^{21}$ addition of sulfur, ${ }^{22}$ or anionic doping. ${ }^{23}$ However, such band gap engineering has only been demonstrated for graphitic carbon nitride $\left(\mathrm{g}-\mathrm{C}_{3} \mathrm{~N}_{4}\right),{ }^{24,25}$ of which the majority narrows the band gap by raising the valence band edge (VBE) so that it is too close to the water oxidation potential to drive water oxidation. It has so far been proved that it is challenging to engineer the band gap of organic photocatalysts by the way of controllable shifting conduction band (CB) positions to enhance the much harder oxygen evolution reaction (with kinetics 5 orders of magnitude slower). ${ }^{16}$ Recently, Wang et al. were successful to prepare a series of CTP organic photocatalysts, with CTP-2 presenting a visible absorption to $530 \mathrm{~nm}$ with a ca. $3 \mu \mathrm{mol} \mathrm{h}$ oxygen evolution rate under full arc irradiation. ${ }^{26}$ Moreover, it is still challenging to demonstrate an organic photocatalyst possessing visible light activity of oxygen evolution comparable to the best inorganic oxygen evolution photocatalysts, for example, $\mathrm{BiVO}_{4}$. The present study employs an efficient strategy to enable the band gap narrowing primarily by shifting down the conduction band edge (CBE) of a wide band gap polymer photocatalyst by oxygenation, not only maintaining the strong oxidation potential but also enhancing visible light absorption to the NIR region; ${ }^{27}$ as such it would be an ideal photocatalyst in a Z-Scheme system for complete water splitting. 1,28

In the past decade, a planar organic material, covalent triazine framework (CT), has been shown to possess promising structural and chemical properties, with a large surface area of ca. $500-2500 \mathrm{~m}^{2} / \mathrm{g}^{27,29-31}$ This material is composed of two molecular units, benzene and triazine, in contrast to the single molecular unit of $\mathrm{g}-\mathrm{C}_{3} \mathrm{~N}_{4}$. Theoretical work demonstrated that this is a semiconductor, with band edges straddling both the oxidation and reduction potentials for water splitting. ${ }^{32}$ Experimentally the onset for light absorption is $2.94 \mathrm{eV}$ due to a very negative CB; light absorption mainly occurs under UV irradiation. ${ }^{33}$ More importantly, band positions of CT are easy to be functionalized through a synthetic process (e.g., by doping), potentially improving the optical absorption and the photocatalytic performance. $^{34}$

By coupling experimental molecular synthesis and simulations, it is identified that oxygen-doped CT (OCT) obtains the modified or disrupted triazine units, which in turn alters optical absorption properties and band gap levels. These new materials composed of carbon, nitrogen, and oxygen show exceptional photocatalytic performance for oxygen generation under visible light, with appreciable external quantum efficiency spanning from UV to $800 \mathrm{~nm}$. Simulation identifies several prospective triazine modifications arising from polymerization in oxygen-containing conditions. In particular, one structure with four distinct carbon sites is proposed, in agreement with all characterizations and the photocatalytic performance of $\mathrm{O}_{2}$ and $\mathrm{H}_{2}$ evolution, experimentally and theoretically, where the doped oxygen results in the suitable unoccupied states in the band gap engineering. Optimum $\mathrm{O}-$ doping effectively narrows the band gap by means of a moderate upshift of the VBE and an adequate downshift of the $\mathrm{CBE}$, resulting in sufficiently large driving forces for hydrogen generation and water oxidation over the whole solar spectrum. Finally, controlling the different amounts of doped oxygen in the frameworks was explored to optimize the band gap and thus enhance the photocatalytic performance of the oxygen evolution.

\section{RESULTS}

We first introduce the three possible oxygenated products applied in the calculations and compared with undoped CT (Figure $1 \mathrm{a}, \mathrm{b}$ ) to explain the general strategy and structural
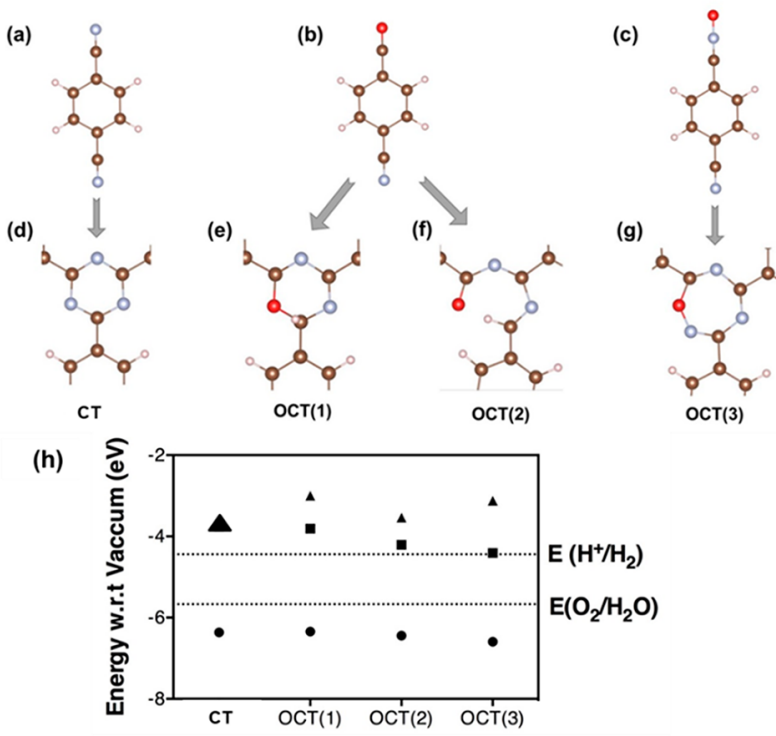

Figure 1. Schematic of formation process of pure CT (a,d), OCT(1) $(b, e), \operatorname{OCT}(2)(b, f)$, and $\operatorname{OCT}(3)(c, g)$ structures. In the ideal process, three dicyanobenzenes coordinate to form triazines. In structure $\mathrm{OCT}(1), \mathrm{O}_{2}$ attacks the nitrile group, replacing nitrogen by oxygen $\left(\mathrm{O}_{\mathrm{N}}\right)$. In structure $\mathrm{OCT}(2)$, the $\mathrm{C}=\mathrm{O}$ bond is created by $\mathrm{O}_{2}$ attacking the nitrile group in the first step and then remains unattacked but emits NO in the second step. In structure OCT(3), one oxygen atom binds to the tail of a nitrile group directly and then forms a single $\mathrm{C}-\mathrm{O}-\mathrm{N}$ linker in the triazine ring. Carbon is represented by a brown sphere, nitrogen gray, hydrogen light pink, and oxygen red. (h) Band alignments of CT and proposed OCT structures. The dots represent the VBEs, and the squares and triangles represent the unoccupied states and CBEs, respectively. The dashed lines are the $\mathrm{H}^{+} / \mathrm{H}_{2}$ (top) and $\mathrm{O}_{2} / \mathrm{H}_{2} \mathrm{O}$ (bottom) redox levels. All the energy is calculated with reference to the vacuum level.

analysis of OCTs. For ideal CT (Figure 1d), the triazine unit forms from condensation of 1,4-dicyanobenzene (Figure 1a) with molten zinc chloride in a low vacuum, following the nucleophile reaction mechanism. The prediction (Figure $1 \mathrm{~b}, \mathrm{c}$ ) was derived from simple tuning on the end of the precursor, 1,4-dicyanobenzene, and preserved the same formation mechanism of the CT. Here CT denotes the structure of the pure triazine ring and those with oxygen-containing structures as $\operatorname{OCT}(1), \operatorname{OCT}(2)$, and $\operatorname{OCT}(3)$ in Figures 1d, 1e, 1f, and $1 \mathrm{~g}$, respectively, with the supercells shown in Figure S1. During the synthesis, oxygen may be used for functionalizing the end groups of the molecules, resulting in the modified triazine and thus modified optical properties. To clarify, oxygen will disrupt the formation of some triazine molecules, forming ketone or nitrous groups in the final materials. The most likely structure, $\operatorname{OCT}(2)$, is discussed later.

OCT was fabricated by copolymerizing monomers of 1,4dicyanobenzene with zinc chloride in a 1:1 molar ratio in the oxygen-containing condition, as shown in Scheme S1. After synthesis, a black crystalline material was observed. The XRD 

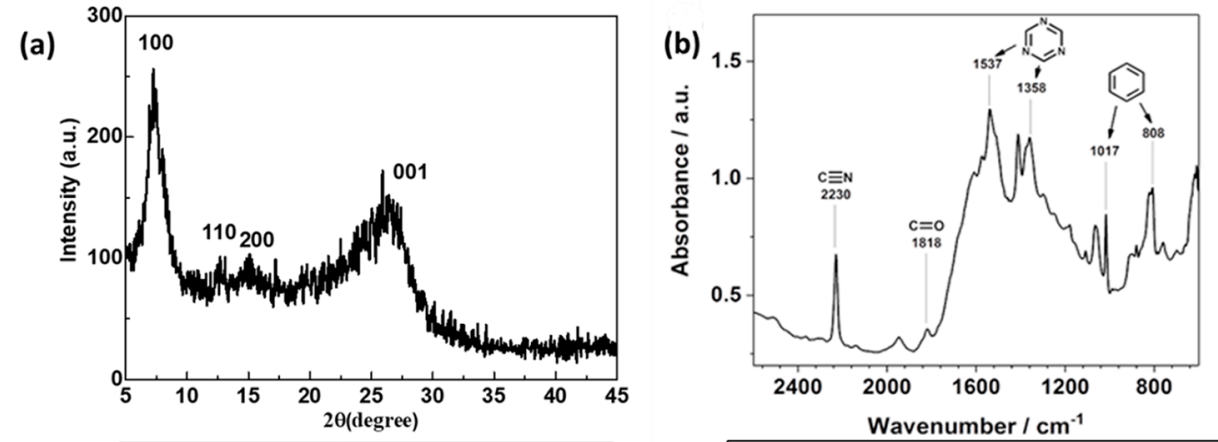

(c)

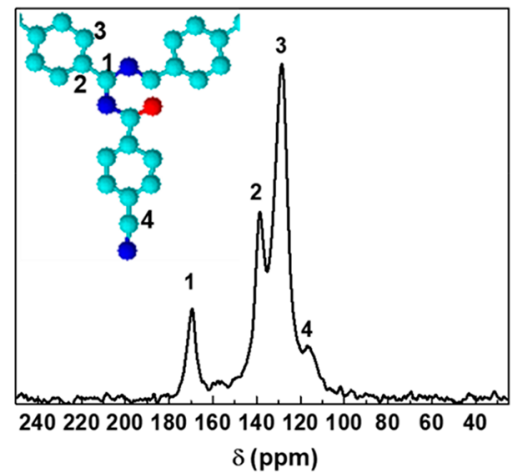

(d)

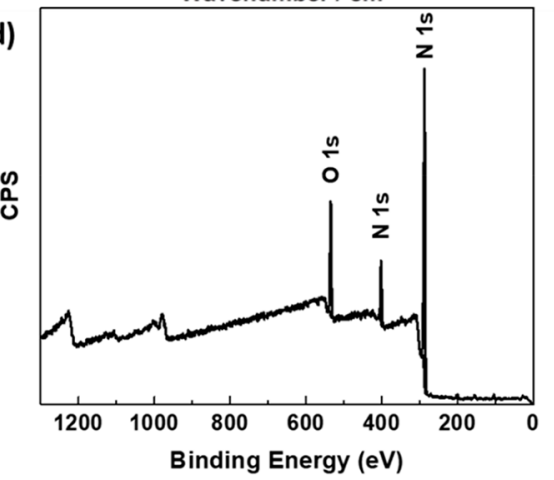

Figure 2. Structure analysis of the 6.68 at. \% OCT. (a) Powder XRD spectra, (b) DRIFTS spectroscopy, (c) ${ }^{13} \mathrm{C}$ cross-polarization magic angle spinning solid-state NMR, and (d) XPS survey spectra after etching to $150 \mathrm{~nm}$ thickness.

analysis shows several intense reflection peaks, namely (100), (110), (200), and (001) in Figure 2a. This indicates that crystalline triazine-based frameworks have been formed. ${ }^{15}$ The large diffraction peak at $2 \theta=26.4^{\circ}$, assigned to the (001) peak, corresponds to an interlayer distance of $3.4 \AA$. Compared with the ideal CT in previous study, ${ }^{30}$ the OCT shares the similar level of crystallinity with the ideal CT but a shift of the (001) peak from $26.1^{\circ}$ to $26.4^{\circ}$. It suggested the stacking distance is slightly reduced due to the doped oxygen atom, which is smaller than the substituted nitrogen atom in the same position. Moreover, from the calculated VBM/CBM (valence band maximum/conduction band minimum) spatial distribution (Figures S2 and S3), the oxygen substitution on the opened triazine units further induces charge delocalization, which can contribute to the van der Waals bonding additionally, and then enhances interlayer integration by reducing the interlayer spacing.

Figure $2 \mathrm{~b}$ shows further structural characterization from DRIFT, which clearly confirmed the existence of triazine units and $\mathrm{C}=\mathrm{O}$ bonds in the OCT in the following details. The peaks at 1017 and $808 \mathrm{~cm}^{-1}$ match well with the $\mathrm{C}=\mathrm{C}$ bending of benzene units ${ }^{35,36}$ and the $\mathrm{C}-\mathrm{H}$ in-plane bending vibrations of the benzene rings, ${ }^{36}$ respectively. The characteristic bonds for the triazine unit were found at $1537 \mathrm{~cm}^{-1}$ (C$\mathrm{N}$ stretching mode) $)^{31,37}$ and $1358 \mathrm{~cm}^{-1}$ (in-plane ring stretching vibrations), ${ }^{37,38}$ confirming the successful formation of triazine rings in our experiments. The peak at $1411 \mathrm{~cm}^{-1}$ corresponds to the absorption by the benzene ring that also forms the basic framework, which proves the existence of the aromatic hydrocarbon. ${ }^{39}$ The peak at $1818 \mathrm{~cm}^{-1}$ represents the $\mathrm{C}=\mathrm{O}$ bond, indicating the presence of oxygen in the polymer. ${ }^{40,41}$ The peak at $2230 \mathrm{~cm}^{-1}$ contributes to the conjugated $\mathrm{C} \equiv \mathrm{N}$ bonds due to the remaining triazine ring terminals. ${ }^{42,43}$
Further structural analysis performed by ${ }^{13} \mathrm{C}$ cross-polarization magic angle spinning (CP-MAS) solid-state NMR (ssNMR) spectroscopy (Figure 2c) shows that the OCT has strong peaks at $170,139,129$, and $116 \mathrm{ppm}$. The peak at 170 ppm was assigned to the symmetric carbon $\mathrm{C} 1$ in the central triazine ring, ${ }^{44}$ while the peaks at 139 and 129 ppm were identified as the two separate carbon atoms of the benzene ring (C2 and C3), respectively. The small peak at $116 \mathrm{ppm}$ was assigned to the terminal nitrile. ${ }^{15,45}$ The $\mathrm{C}=\mathrm{O}$ band signal is at $174 \mathrm{ppm}$, dominated by the symmetric carbon $\mathrm{C} 1$ signal and overlapping with the peak at $170 \mathrm{ppm}$. The elemental composition analysis was made by XPS in Figure $2 \mathrm{~d}$. The presence of oxygen is detected with no $\mathrm{ZnCl}$ residue left in the samples. Detailed analysis of the OCT C 1 s and $\mathrm{O} 1 \mathrm{~s}$ signal profiles (Figure S4) reveals $\mathrm{C}=\mathrm{O}$ peaks at both $288.0 \mathrm{eV}$ in $\mathrm{C}$ $1 \mathrm{~s}$ XPS spectra and $533.0 \mathrm{eV}$ in O $1 \mathrm{~s}$ XPS spectra. This agrees with the FTIR spectra that show carbon-oxygen double bonds. EELS measurements were further taken from numerous positions in thin sections of the sample, which indicate the presence of oxygen in Figure S5. To obtain a higher signal-tonoise $\mathrm{C}, \mathrm{N}$, and $\mathrm{O}$ K-edge EELS, we averaged three EELS measurements taken in different regions of sample, shown in Figure S5d, where the ADF-STEM image of line profiles across the edge of sample were taken. The reason to collect data from the thin edge of sample is to make sure the beam passes through the samples and the outlet signal can be collected. Figures S5a, S5b, and S5c show the C, N, and O K-edge EELS signal, respectively, from the line in Figure S5d. Analysis of C and $\mathrm{N}$ K-edge EELS shows that the system is practically exclusively composed of $\mathrm{sp}^{2}$-hybridized carbon and nitrogen atoms, as indicated by the presence of the $1 \mathrm{~s} \rightarrow \pi^{*}$ transition for both elements. The carbon-K ionization edge and nitrogen$\mathrm{K}$ ionization edge show identical near-edge structures, indicating a similar 3-fold coordination and electronic environment of carbon and nitrogen in the synthesized 
(a)

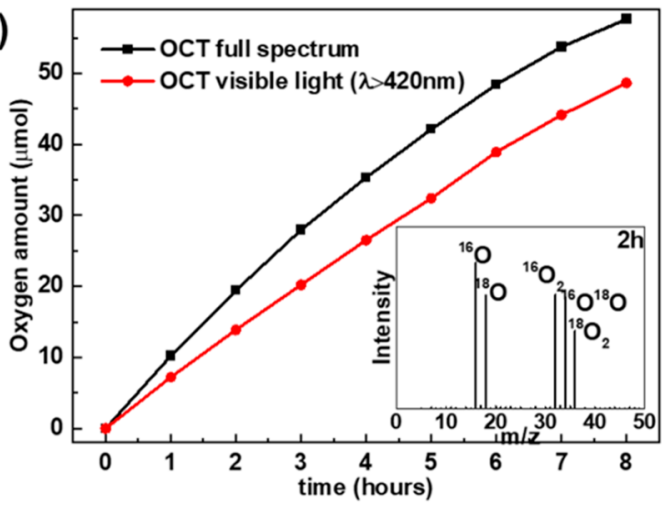

(c)

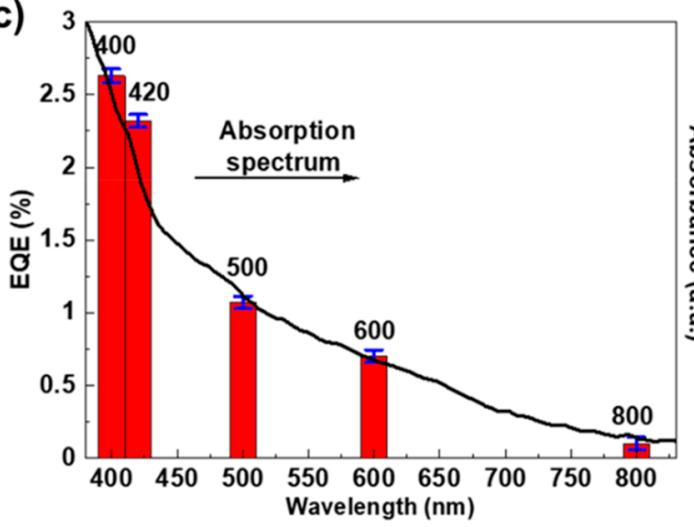

(b)

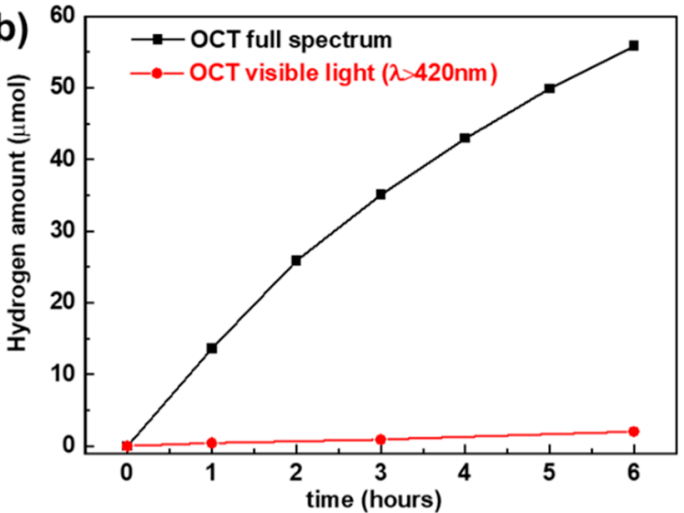

(d)

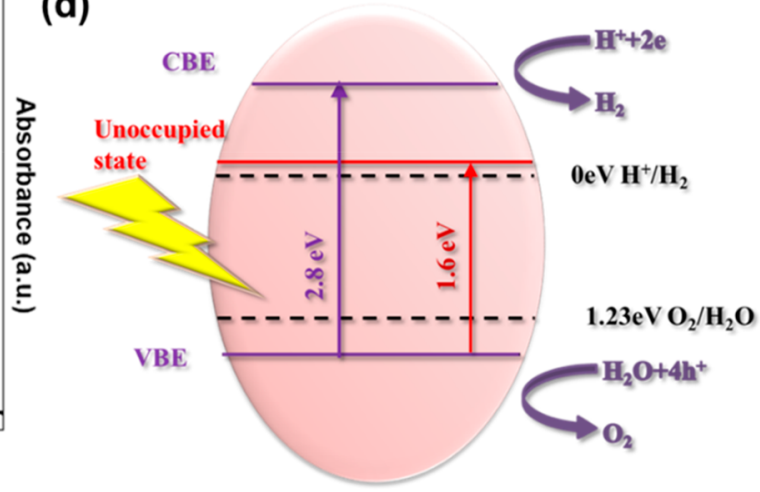

Figure 3. Photocatalytic performance and band structure diagram of 6.68 at. \% OCT. Time courses for oxygen (a) and hydrogen (b) production from water under full arc irradiation and visible light illumination $(\lambda>420 \mathrm{~nm})$ in the presence of relevant scavengers and (a) an inset of gas chromatography mass spectra (GC-MS) of oxygen obtained under full arc irradiation in the $95 \%{ }^{18} \mathrm{O}$-labeled-water photocatalytic system after $2 \mathrm{~h}$ reaction. (c) External quantum efficiency at 400, 420, 500, 600, and $800 \mathrm{~nm}$ (left-hand axis) with an optical absorption spectrum plotted (righthand side). (d) Band positions and the pathways for water redox of 6.68 at. \% OCT, where the CBE is the original conduction band in the unmodified CT and the unoccupied state is the lowered CB in the OCT.

material. ${ }^{46}$ The first peak of $\mathrm{O}$ K-edge EELS spectrum at 532 $\mathrm{eV}$ is assigned to $\mathrm{O}_{\mathrm{C}=\mathrm{O}} 1 \mathrm{~s} \rightarrow \pi^{* 47}$ in Figure $\mathrm{S5c}$, and the second peak at $538 \mathrm{eV}$ is attributed to $\mathrm{O}_{\mathrm{C}=\mathrm{O}} 1 \mathrm{~s} \rightarrow 3 \mathrm{p}$ according to the EELS study of liquid acetone, ${ }^{46,48}$ which strongly indicates the presence of the $\mathrm{C}=\mathrm{O}$ structure in the materials. Elemental analysis (EA) shows that the precise molar ratio of $\mathrm{C}$ to $\mathrm{N}$ is around 4.5 , as detailed in Table S1, which is closed to the theoretical value of 4 for the pure CT. ${ }^{30}$ The 6.68 at. \% oxygen gradient into the sample may be due to initial rapid nucleation and seed formation of CT with insufficient oxygen, and then slow growth/polymerization occurred, allowing the incorporation of relatively high levels of oxygen. The sample has a large BET surface area of $388.7 \mathrm{~m}^{2} / \mathrm{g}$ with a pore size of $0.212 \mathrm{~cm}^{3} / \mathrm{g}$, similar to other values obtained experimentally. ${ }^{31}$ Above all, oxygen-doped covalent triazine materials were synthesized successfully by the creation of $\mathrm{C}=\mathrm{O}$ bonds in the structure (structure OCT(2)), which can play a key role in optical, electronic, and photocatalytic properties as discussed in the following.

Density functional theory (DFT) study was taken into consideration based on the formation process (see Figure 1) and probed the electronic structures and optical properties of several prospective modified structures.

To compare the pure CT and the OCTs, the reaction energy was calculated according to the following equation: $E_{\mathrm{f}}=$ $E_{\mathrm{CT} / \mathrm{OCTs}}-2 E_{\text {precursor }, 0}-E_{\text {precursor }}+\Delta E_{\mathrm{H}}$, where the $E_{\mathrm{CT} / \mathrm{OCTs}}$ is the total energy of the systems of CT or OCTs. The $E_{\text {precursor, } 0}$ represents the total energy of the 1,4-dicyanobenzene molecule, and $E_{\text {precursor }}$ is the energy of the proposed precursors shown in Figure $1 \mathrm{~b}, \mathrm{c}$. The $\Delta E_{\mathrm{H}}$ is the adjustment energy to balance the equation and was calculated by half of the energy of $\mathrm{H}_{2}$. The reaction energy of each structure is listed in Table S2. From the thermodynamic point of view, all the reactions are exothermic; so, all the three structures could be produced in the final compounds. Structures OCT(1) and OCT(3) conserve the framework, while OCT(2) disrupts the triazine unit that links benzenes, hindering complete crystallization of the OCT and resulting in crystal disorder as observed in XRD (Figure 2a).

The oxygenation not only disturbs the formation of the triazine ring but, more importantly, also changes the electronic distribution on VBM and CBM, as shown in Figure S2. For the pure CT (Figure S2a,b), the nitrogen atoms contribute to both VBM and CBM, respectively, by electrons from $\mathrm{p}_{x}$ and $\mathrm{p}_{y}$ orbitals and from $\mathrm{p}_{z}$ orbitals. The oxygen doping dramatically tunes the configuration of the band configurations. For the OCT(1), both carbon and oxygen donate $\mathrm{p}_{z}$ electrons to the VBM. For OCT(2) and OCT(3), the VBM is from electrons over a wider area, especially in the carbonyl group. Moreover, the oxygen does not contribute to the CBM in OCT(1) and OCT(3) but does partially to the CBM from the $\mathrm{p}_{z}$ orbital in OCT(2). Overall, partially distorted triazine units due to oxygenation show relatively high delocalization of electron density (Figure S2), implying that photogenerated charges are more ready to diffuse through the OCT to react with other species than through the pure CT. Thus, oxygen incorporation 


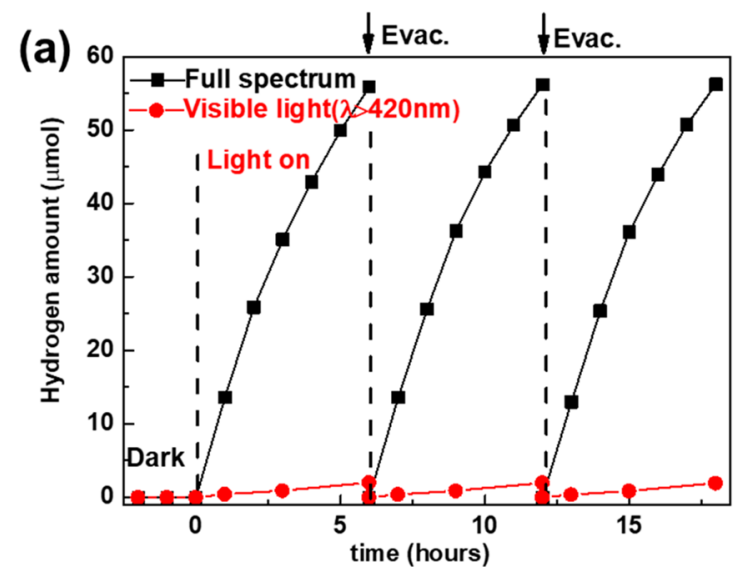

(b)

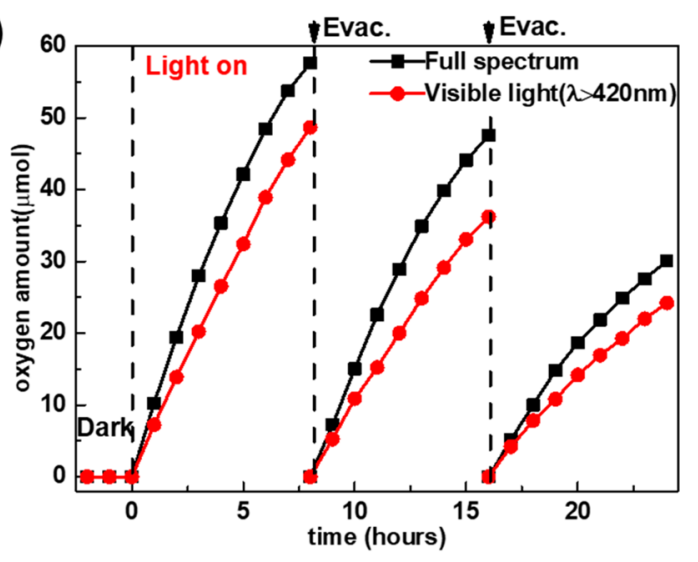

Figure 4. Typical time courses of $(\mathrm{a}) \mathrm{H}_{2}$ production from water containing 10 vol \% TEOA as an electron donor and 3 wt $\% \mathrm{Pt}\left(\mathrm{H}_{2} \mathrm{PtCl} \mathrm{l}_{6}\right)$ as a cocatalyst and (b) $\mathrm{O}_{2}$ production from water containing $1 \mathrm{~g}$ of $\mathrm{AgNO}_{3}$ as a hole scavenger, under full arc and visible light (of wavelength longer than $420 \mathrm{~nm}$ ) by 6.68 at. \% OCT. The reaction of $\mathrm{O}_{2}$ evolution was run for $24 \mathrm{~h}$, with purge by $\mathrm{Ar}$ every $8 \mathrm{~h}$ (dashed line). The reaction of $\mathrm{H}_{2}$ evolution was run for $18 \mathrm{~h}$, with purge by Ar every $6 \mathrm{~h}$ (dashed line). Continuous $\mathrm{O}_{2}$ is produced but with the somewhat degradation from the beginning of second cycle, which is reasonable due to the deposition of metallic silver on the catalyst surface when using $\mathrm{Ag}^{+}$as an electron scavenger, blocking light absorption and obstructing active sites. The overall water splitting for both $\mathrm{H}_{2}$ and $\mathrm{O}_{2}$ production simultaneously from water without any sacrificial agents was observed under full arc irradiation as shown in Figure S11, which indicates the possibility that the ratio of $\mathrm{H}_{2}$ to $\mathrm{O}_{2}$ is far from the ideal. A further optimization of the cocatalysts is underway.

would dramatically narrow the band gap and is also supposed to inhibit the recombination of electrons and holes. ${ }^{49}$

The photocatalytic activity of the 6.68 at. \% OCT was determined in the presence of sacrificial reagents under full arc and visible irradiation in Figure 3. Without cocatalysts, the OCT shows near-linear oxygen evolution from the aqueous solution with an initial rate of $10.24 \mu \mathrm{mol} \mathrm{h}^{-1}$ (Figure 3a). Remarkably, the photocatalytic activity for oxygen production under visible light $(\lambda>420 \mathrm{~nm})$ is very close to that under full arc, distinguished from the water oxidation photocatalysts reported so far, where visible-light-driven activity is always much smaller than full-arc-driven activity. We also investigated the ability of the OCT to produce hydrogen, with triethanolamine as an electron donor and $\mathrm{Pt}$ as a cocatalyst. The OCT produces hydrogen from water at an average rate of ca. 13 $\mu \mathrm{mol} \mathrm{h}{ }^{-1}$ under full arc irradiation (Figure $3 \mathrm{~b}$ ). Under visible light, hydrogen is produced at ca. $0.5 \mu \mathrm{mol} \mathrm{h}^{-1}$. This suggests that there are likely at least two low-energy accessible unoccupied states in the OCT, one significantly more positive than the other. The more positive unoccupied state is unable to provide enough driving force for the proton reduction, hindering hydrogen production under visible light illumination as shown in Figure $3 \mathrm{~d}$. The experimental measurement obtained from the UV-vis absorption data (Figure $3 c$ and Figure S6) determined two optical band gaps with separation ca. 1.6 and $2.8 \mathrm{eV}$. Valence band X-ray photoelectron spectra were measured for $\mathrm{g}-\mathrm{C}_{3} \mathrm{~N}_{4}$ and OCT (Figure S7). The valence band of OCT is $0.3 \mathrm{eV}$ more negative than that of $\mathrm{g}-\mathrm{C}_{3} \mathrm{~N}_{4}$. The valence band of $\mathrm{g}_{-} \mathrm{C}_{3} \mathrm{~N}_{4}$ was reported as ca. $+1.8 \mathrm{eV}{ }^{49}$ Thus, these experimental data confirmed the VBM of $+1.5 \mathrm{eV}$ and two CBM of -0.1 and $-1.3 \mathrm{eV}$, consistent with the modeling results. The modified polymer photocatalyst shows a very attractive activity beyond $600 \mathrm{~nm}$, more competitive than the majority of inorganic photocatalysts. Under full arc irradiation, the electrons are excited from the VBE to the unoccupied state and $\mathrm{CBE}$, enabling both efficient $\mathrm{O}_{2}$ and $\mathrm{H}_{2}$ productions. Under visible light irradiation, electrons are excited from the VBE only to the unoccupied state, so the rate of oxygen generation is maintained, but the rate of hydrogen production is relatively slow due to the reduced driving force for the hydrogen evolution. The feature that appears near $750 \mathrm{~nm}$ is assigned to $\mathrm{n}-\pi^{*}$ transitions involving lone pairs on the doped $\mathrm{O}$ atoms within the layers to the $\pi^{*}$ levels of the condensed rings, as shown in Figure S2. Thus, the unoccupied state with contributions from the $\mathrm{p}_{z}$ orbitals includes the $\sigma$ and $\sigma^{*} \mathrm{C}-\mathrm{N}$ bonds and the lone pairs on the oxygen atoms in the distorted triazine rings, as shown in Figure S2f. The bottom of the conduction band CBM is instead formed by $\mathrm{p}_{z}$ contributions on the $\mathrm{C}$ and $\mathrm{N}$ atoms of the triazine rings and has therefore a level of $\pi^{*}$ character, as shown in Figure S2b. This results in the intense band of $2.8 \mathrm{eV}$ and is assigned to $\pi-\pi^{*}$ transitions that are commonly observed in conjugated ring systems including heterocyclic aromatics. ${ }^{50,51}$ As a result, we believe there are two band gaps in the OCT, and these correlate well with the photocatalytic activity and experimental data.

To determine the efficiency for oxygen generation of the OCT, we compared to one water oxidation photocatalyst the monoclinic $\mathrm{BiVO}_{4}$, prepared by an aqueous process using layered compounds. ${ }^{52,53}$ From inspection in Figure S8, the OCT is as efficient as $\mathrm{BiVO}_{4}\left(8.29 \mu \mathrm{mol} \mathrm{h}^{-1}\right)$ when a $420 \mathrm{~nm}$ cutoff filter was used. Figure $3 \mathrm{c}$ shows the external quantum efficiency (EQE) for oxygen production, which is more reliable as an indicator of photocatalyst efficiency. Notably, compared with the nearly pure $\mathrm{CT}^{54}$ and CTF- $0,{ }^{34}$ the OCT has a much wider operation window, with an EQE of $2.6 \%$ at $400 \mathrm{~nm}$, $1.1 \%$ at $500 \mathrm{~nm}$, and even $0.2 \%$ at wavelengths as long as 800 $\mathrm{nm}$, while the previous polymers show the oxygen production only when using $3 \mathrm{wt} \% \mathrm{RuO}_{x}$ as a cocatalyst below $600 \mathrm{~nm}^{54}$ The OCT has obtained the large amount of $\mathrm{O}_{2}$ but no need of any noble-metal cocatalysts, also 5 times higher than the reported CTP-2 under the similar reaction condition. ${ }^{26}$ Moreover, the previous one exhibited the relatively low water oxidation activity at long wavelength even by decorating certain cocatalysts, for example, an AQY of $0.2 \%$ at $600 \mathrm{~nm}^{.54}$ However, the OCT shows the strong water oxidation ability at $600 \mathrm{~nm}$ without any cocatalysts, for example, the EQE of $1 \%$. Such a long wavelength response goes far beyond any inorganic and organic photocatalysts reported so far for 

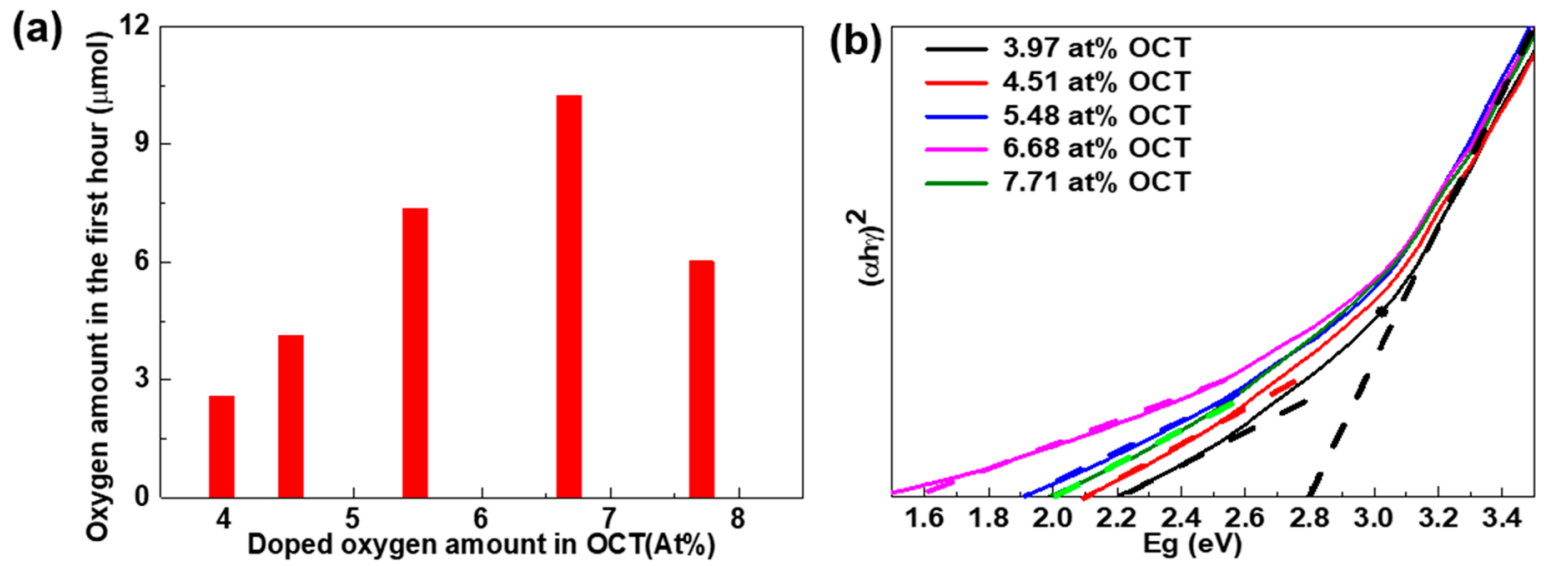

Figure 5. Oxygen evolution amount for different amounts of oxygen doped in OCT (a) and the corresponding Tauc plots calculated based on the UV-vis spectra (b).

oxygen evolution. In addition, the correlation between UV-vis absorption spectrum and EQE suggests that water oxidation is driven by light.

Finally, we assessed the stability of our samples by running a three-cycle photocatalytic test, undertaking structural analysis before and after water oxidation and hydrogen evolution. Obviously, as shown in Figure 4a, the reaction of $\mathrm{H}_{2}$ evolution was run for a total of $18 \mathrm{~h}$ with intermittent purging by $\mathrm{Ar}$ every $6 \mathrm{~h}$ under both full arc and visible light irradiation $(\lambda>$ $420 \mathrm{~nm})$. The amount of $\mathrm{H}_{2}$ evolution is almost the same at the end of every cycle, which means the stability of 6.68 at. \% OCT during the reaction. On the other hand, the reaction of $\mathrm{O}_{2}$ evolution was run for a total of $24 \mathrm{~h}$ within intermittent purging by Ar every $8 \mathrm{~h}$ under both full arc and visible light irradiation (Figure 4b), whereas the structure of photocatalysts remains stable after reaction, as confirmed by the FTIR and ssNMR spectra taken before and after water oxidation in Figure S9. Both demonstrate the stability of the polymer during photocatalytic reaction.

We further verified the detected $\mathrm{O}_{2}$ was indeed generated by water oxidation. First, the total evolution amount of $\mathrm{O}_{2}$ obtained after $24 \mathrm{~h}$ was ca. $135.2 \mu \mathrm{mol}$ of $\mathrm{O}_{2}$ or $270.4 \mu \mathrm{mol}$ of oxygen atoms, as shown in Figure 4a. This is more than 4 times the amount of oxygen atoms $(63.3 \mu \mathrm{mol})$ contained in the 6.68 at. \% OCT sample drawn from the elemental analysis in Table S2, proving that the oxygen gas was produced from water rather than deoxygenation of the polymer. In addition, isotopic measurement showed that the photocatalytic oxygen evolution in the ${ }^{18} \mathrm{O}$-labeled water generates a strong ${ }^{18} \mathrm{O}_{2}$ peak after reaction for $2 \mathrm{~h}$ reaction, as noted in the GC-MS spectra in Figure S10 and the inset in Figure 3a, further confirming that the oxygen production is originally from the water.

We next compared the density of states (DOS) of CT and OCTs in Figure S3. The band gap of the pure CT is $2.6 \mathrm{eV}$, and $\operatorname{OCT}(1)$ shows a slight reduction in band gap, to $2.54 \mathrm{eV}$, whereas the other structures display significant red-shifts in absorption by $0.37 \mathrm{eV}$ (OCT(2)) and $0.42 \mathrm{eV}$ (OCT(3)). The unoccupied states result from the formation of the oxygencontaining functional groups, albeit with significant delocalization arising from the significant aromaticity of the benzene and triazine components of the CT. As shown in Figure 1h, oxygenation of the polymer dramatically reduces the overpotential for $\mathrm{H}_{2}$ production under visible light irradiation but almost retains a relatively stable $\mathrm{VBE}$, not affecting $\mathrm{O}_{2}$ production. Among them in Figure 1h, the $\operatorname{OCT}(2)$ demonstrates the narrowest band gap, most benefitting the visible optical absorption, but the unoccupied state is too close to the potential of water reduction to produce $\mathrm{H}_{2}$ under visible light irradiation; thus, efficient hydrogen production only occurs under full arc irradiation. Furthermore, $\mathrm{OCT}(2)$ is the only structure containing the $\mathrm{C}=\mathrm{O}$ bonding, which is consistent with the experimental structure characterization results and further confirms that $\mathrm{OCT}(2)$ is the most promising structure matching all the characterization and simulation results.

Furthermore, we explored the effect of oxygen content in the materials on their water oxidation performance. Five samples with different levels of oxygen doping were synthesized, and the detailed elemental ratios are shown in Table $S 1$. The $\mathrm{C} / \mathrm{N}$ ratios of all polymers are around 4.5 , close to the theoretical value of 4 for the pure CT. The slightly higher ratio is believed to be mainly due to oxygen doping and more benzene ring terminals or edges. The higher the level of oxygen doped in the framework, the narrower the band gap, up to $1.6 \mathrm{eV}$ when an oxygen content is 6.68 at. \% (Figure $5 b$ ). Moreover, the effect of the level of doped oxygen on the photocatalytic performance and the band gap conversed from UV-vis absorption has been further investigated experimentally, shown in Figure 5a. The results show that the amount of photocatalytic oxygen evolution increases as the oxygen doping level increases to a peak at the oxygen-doping level of 6.68 at. \%, of the initial rate of $10.24 \mu \mathrm{mol}$. Afterward, the amount of produced oxygen drops as the oxygen doping level increases to 7.71 at. \%. The trend of this experimental performance is in good agreement with the changes of calculated the band positions and the band gap of the OCT polymers, as shown in Figure S12. Compared to the pure CT, the unoccupied state of OCT shifts down greatly, and the VB relatively remains, thus narrowing the band gap. Above all, doping oxygen into covalent triazine framework synthesized by a controlled polymerization method realized readily band gap engineering. The UV-vis absorption spectra of different oxygen-incorporated CT polymers show the increase in the level of doped oxygen, leading to a long wavelength absorption value, $\lambda_{\max }$ due to a narrower band gap at a higher level of doped-oxygen in the $\mathrm{CT}$, as predicted theoretically. With further increase of the oxygen doping level, as indicated by the XPS spectra in Figure S13, the extra oxygen tends to form $\mathrm{C}-\mathrm{O} / \mathrm{C}-\mathrm{OH}$ bonds, rather than the $\mathrm{C}=\mathrm{O}$ bonds. This indicates that the $\mathrm{C}=\mathrm{O}$ bond is more effective to 
tune the band gap and, more importantly, acts as an active site for water oxidation.

\section{CONCLUSIONS}

In conclusion, we have successfully synthesized an oxygendoped covalent triazine (OCT) material by polymerization of 1,4-dicyanobenzene in controlled gas mixtures of oxygen and nitrogen. Under full arc irradiation, the new OCT photocatalyst shows both oxygen and hydrogen evolutions from water in the presence of separate scavengers and demonstrates oxygen evolution longer than a day. For the first time, water oxidation proceeds from UV up to $800 \mathrm{~nm}$ irradiation by a metal-free photocatalyst, consistent with the UV-vis absorption spectrum, with an EQE of close to $1 \%$ even at $600 \mathrm{~nm}$. The visible light absorption is attributed to a significant reduction in the gap, caused by disruption of the triazine structural unit and the formation of the effective $\mathrm{C}=\mathrm{O}$ moiety. This moiety has a very significant effect on the band gap engineering, creating the unoccupied states, which benefits the water oxidation under the visible light irradiation. We have demonstrated, for the first time, an organic water oxidation photocatalyst that has an exceptionally wide window of operation, up to $800 \mathrm{~nm}$. Overall, our findings demonstrate a strong potential for the development of low-cost and environmental-friendly photocatalysts for effective solar energy conversion and environmental remediation.

\section{ASSOCIATED CONTENT}

\section{SI Supporting Information}

The Supporting Information is available free of charge at https://pubs.acs.org/doi/10.1021/acsaem.0c01153.

Experimental methods, photocatalytic performances, details and results of computational simulations, XPS spectra, EELS results, ADF-STEM image, elemental analysis results, optical absorption spectra, FTIR spectra, gas chromatography mass spectra (PDF)

\section{AUTHOR INFORMATION}

\section{Corresponding Authors}

Junwang Tang - Department of Chemical Engineering University College London, London WC1E 7JE, U.K.; 이이.org/0000-0002-2323-5510; Email: junwang.tang@ ucl.ac.uk

Zheng-Xiao Guo - Department of Chemistry, University College

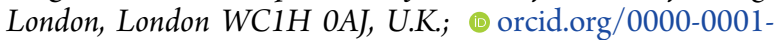
5404-3215; Email: z.x.guo@ucl.ac.uk

\section{Authors}

Dan Kong - Department of Chemical Engineering, University College London, London WC1E 7JE, U.K.; 우 orcid.org/00000003-2523-9037

Xiaoyu Han - Department of Chemistry, University College London, London WC1H 0AJ, U.K.

Stephen A. Shevlin - Department of Chemistry, University

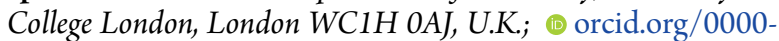
0001-5896-0301

Christopher Windle - Department of Chemical Engineering, University College London, London WC1E 7JE, U.K.; (1) orcid.org/0000-0002-7956-7063

Jamie H. Warner - Department of Materials, University of Oxford, Oxford OX1 3PH, U.K.; (1) orcid.org/0000-00021271-2019
Complete contact information is available at: https://pubs.acs.org/10.1021/acsaem.0c01153

\section{Notes}

The authors declare no competing financial interest.

\section{ACKNOWLEDGMENTS}

Financial contributions from the following sources are gratefully acknowledged: UCL Engineering Dean's Price and the Chinese Scholarship Council (CSC) for a PhD studentship (D.K.), the European Community Seventh Framework Programmes (4G-PHOTOCAT 309636 and NANOSIM 604656), the EPSRC (EP/K021192/1, EP/S018204/2), the Leverhulme Trust (RPG-2017-122), and Royal SocietyNewton Advanced Fellowship grant (NAF $\backslash$ R $1 \backslash 191163$ and NA170422). Computational work was performed using the UCL Grace High Performance Computing Facilities (Grace@ UCL), with additional compute support from the ARCHER National Computing Facility by a Resource Allocation Project (Project e454). D.K. also thanks Drs. Hasmukh Patel, Kaipei Qiu, and Srinivas Gadipelli for tube furnace training, Dr. Mustafa Bayazit for TEM observation, and Chiching Lau for BET training. S.A.S. thanks Dr. Guoliang Chai for useful discussions.

\section{REFERENCES}

(1) Kong, D.; Zheng, Y.; Kobielusz, M.; Wang, Y.; Bai, Z.; Macyk, W.; Wang, X.; Tang, J. Recent advances in visible light-driven water oxidation and reduction in suspension systems. Mater. Today 2018, 21 (8), 897-924.

(2) Moniz, S. J. A.; Shevlin, S. A.; Martin, D. J.; Guo, Z.-X.; Tang, J. Visible-light driven heterojunction photocatalysts for water splitting a critical review. Energy Environ. Sci. 2015, 8 (3), 731-759.

(3) Muhich, C. L.; Ehrhart, B. D.; Al-Shankiti, I.; Ward, B. J.; Musgrave, C. B.; Weimer, A. W. A review and perspective of efficient hydrogen generation via solar thermal water splitting. Wiley Interdisciplinary Reviews: Energy and Environment, 2015.

(4) Hisatomi, T.; Kubota, J.; Domen, K. Recent advances in semiconductors for photocatalytic and photoelectrochemical water splitting. Chem. Soc. Rev. 2014, 43 (22), 7520-7535.

(5) Osterloh, F. E. Inorganic nanostructures for photoelectrochemical and photocatalytic water splitting. Chem. Soc. Rev. 2013, 42 (6), 2294-2320

(6) Tachibana, Y.; Vayssieres, L.; Durrant, J. R. Artificial photosynthesis for solar water-splitting. Nat. Photonics 2012, 6 (8), 511-518.

(7) Zhuang, H. L.; Hennig, R. G. Single-Layer Group-III Monochalcogenide Photocatalysts for Water Splitting. Chem. Mater. 2013, 25 (15), 3232-3238.

(8) Jiang, C.; Moniz, S. J. A.; Khraisheh, M.; Tang, J. EarthAbundant Oxygen Evolution Catalysts Coupled onto ZnO Nanowire Arrays for Efficient Photoelectrochemical Water Cleavage. Chem. Eur. J. 2014, 20 (40), 12954-12961.

(9) Sandroni, M.; Gueret, R.; Wegner, K. D.; Reiss, P.; Fortage, J.; Aldakov, D.; Collomb, M. N. Cadmium-free CuInS2/ZnS quantum dots as efficient and robust photosensitizers in combination with a molecular catalyst for visible light-driven $\mathrm{H}_{2}$ production in water. Energy Environ. Sci. 2018, 11 (7), 1752-1761.

(10) Xie, G.; Zhang, K.; Guo, B.; Liu, Q.; Fang, L.; Gong, J. R. Graphene-Based Materials for Hydrogen Generation from LightDriven Water Splitting. Adv. Mater. 2013, 25 (28), 3820-3839.

(11) Wang, X.; Maeda, K.; Thomas, A.; Takanabe, K.; Xin, G.; Carlsson, J. M.; Domen, K.; Antonietti, M. A metal-free polymeric photocatalyst for hydrogen production from water under visible light. Nat. Mater. 2009, 8 (1), 76-80.

(12) Martin, D. J.; Qiu, K.; Shevlin, S. A.; Handoko, A. D.; Chen, X.; Guo, Z.; Tang, J. Highly Efficient Photocatalytic H2 Evolution from 
Water using Visible Light and Structure-Controlled Graphitic Carbon Nitride. Angew. Chem., Int. Ed. 2014, 53 (35), 9240-9245.

(13) Sprick, R. S.; Bonillo, B.; Clowes, R.; Guiglion, P.; Brownbill, N. J.; Slater, B. J.; Blanc, F.; Zwijnenburg, M. A.; Adams, D. J.; Cooper, A. I. Visible-Light-Driven Hydrogen Evolution Using Planarized Conjugated Polymer Photocatalysts. Angew. Chem., Int. Ed. 2016, 55 (5), 1792-1796.

(14) Wang, K.; Yang, L.-M.; Wang, X.; Guo, L.; Cheng, G.; Zhang, C.; Jin, S.; Tan, B.; Cooper, A. Covalent Triazine Frameworks via a Low-Temperature Polycondensation Approach. Angew. Chem., Int. Ed. 2017, 56 (45), 14149-14153.

(15) Schwinghammer, K.; Hug, S.; Mesch, M. B.; Senker, J.; Lotsch, B. V. Phenyl-Triazine Oligomers for Light-Driven Hydrogen Evolution. Energy Environ. Sci. 2015, 8, 3345-3353.

(16) Tang, J.; Durrant, J. R.; Klug, D. R. Mechanism of Photocatalytic Water Splitting in $\mathrm{TiO}_{2}$. Reaction of Water with Photoholes, Importance of Charge Carrier Dynamics, and Evidence for Four-Hole Chemistry. J. Am. Chem. Soc. 2008, 130 (42), 1388513891.

(17) Yi, Z.; Ye, J.; Kikugawa, N.; Kako, T.; Ouyang, S.; StuartWilliams, H.; Yang, H.; Cao, J.; Luo, W.; Li, Z.; Liu, Y.; Withers, R. L. An orthophosphate semiconductor with photooxidation properties under visible-light irradiation. Nat. Mater. 2010, 9 (7), 559-564.

(18) Wang, Q.; Hisatomi, T.; Jia, Q.; Tokudome, H.; Zhong, M.; Wang, C.; Pan, Z.; Takata, T.; Nakabayashi, M.; Shibata, N.; Li, Y.; Sharp, I. D.; Kudo, A.; Yamada, T.; Domen, K. Scalable water splitting on particulate photocatalyst sheets with a solar-to-hydrogen energy conversion efficiency exceeding 1\%. Nat. Mater. 2016, 15 (6), 611615.

(19) Li, Y.; Zhu, J.; Chu, H.; Wei, J.; Liu, F.; Lv, M.; Tang, J.; Zhang, B.; Yao, J.; Huo, Z.; Hu, L.; Dai, S. $\mathrm{BiVO}_{4}$ semiconductor sensitized solar cells. Sci. China: Chem. 2015, 58 (9), 1489-1493.

(20) Martin, D. J.; Liu, G.; Moniz, S. J. A.; Bi, Y.; Beale, A. M.; Ye, J.; Tang, J. Efficient visible driven photocatalyst, silver phosphate: performance, understanding and perspective. Chem. Soc. Rev. 2015, 44 (21), 7808-7828.

(21) Zhang, J. S.; Zhang, G. G.; Chen, X. F.; Lin, S.; Mohlmann, L.; Dolega, G.; Lipner, G.; Antonietti, M.; Blechert, S.; Wang, X. C. CoMonomer Control of Carbon Nitride Semiconductors to Optimize Hydrogen Evolution with Visible Light. Angew. Chem., Int. Ed. 2012, 51 (13), 3183-3187.

(22) Zhang, J. S.; Sun, J. H.; Maeda, K.; Domen, K.; Liu, P.; Antonietti, M.; Fu, X. Z.; Wang, X. C. Sulfur-mediated synthesis of carbon nitride: Band-gap engineering and improved functions for photocatalysis. Energy Environ. Sci. 2011, 4 (3), 675-678.

(23) Shevlin, S. A.; Guo, Z. X. Anionic Dopants for Improved Optical Absorption and Enhanced Photocatalytic Hydrogen Production in Graphitic Carbon Nitride. Chem. Mater. 2016, 28 (20), 72507256.

(24) Liu, J.; Liu, Y.; Liu, N. Y.; Han, Y. Z.; Zhang, X.; Huang, H.; Lifshitz, Y.; Lee, S. T.; Zhong, J.; Kang, Z. H. Metal-free efficient photocatalyst for stable visible water splitting via a two-electron pathway. Science 2015, 347 (6225), 970-974.

(25) Wang, Y.; Bayazit, M. K.; Moniz, S. J. A.; Ruan, Q.; Lau, C. C.; Martsinovich, N.; Tang, J. Linker-controlled polymeric photocatalyst for highly efficient hydrogen evolution from water. Energy Environ. Sci. 2017, 10 (7), 1643-1651.

(26) Lan, Z. A.; Fang, Y.; Zhang, Y.; Wang, X. Photocatalytic Oxygen Evolution from Functional Triazine-Based Polymers with Tunable Band Structures. Angew. Chem., Int. Ed. 2018, 57 (2), 470474.

(27) Wang, P.; Jiang, X.; Zhao, J. Bottom-up design of 2D organic photocatalysts for visible-light driven hydrogen evolution. J. Phys.: Condens. Matter 2016, 28 (3), 034004.

(28) Wang, Y.; Suzuki, H.; Xie, J.; Tomita, O.; Martin, D. J.; Higashi, M.; Kong, D.; Abe, R.; Tang, J. Mimicking Natural Photosynthesis: Solar to Renewable H2 Fuel Synthesis by Z-Scheme Water Splitting Systems. Chem. Rev. 2018, 118 (10), 5201-5241.
(29) Katekomol, P.; Roeser, J.; Bojdys, M.; Weber, J.; Thomas, A. Covalent Triazine Frameworks Prepared from 1,3,5-Tricyanobenzene. Chem. Mater. 2013, 25 (9), 1542-1548.

(30) Kuhn, P.; Antonietti, M.; Thomas, A. Porous, Covalent Triazine-Based Frameworks Prepared by Ionothermal Synthesis. Angew. Chem., Int. Ed. 2008, 47 (18), 3450-3453.

(31) Bojdys, M. J.; Jeromenok, J.; Thomas, A.; Antonietti, M. Rational Extension of the Family of Layered, Covalent, TriazineBased Frameworks with Regular Porosity. Adv. Mater. 2010, 22 (19), 2202-2205.

(32) Jiang, X.; Wang, P.; Zhao, J. 2D covalent triazine framework: a new class of organic photocatalyst for water splitting. J. Mater. Chem. A 2015, 3 (15), 7750-7758.

(33) Bi, J.; Fang, W.; Li, L.; Wang, J.; Liang, S.; He, Y.; Liu, M.; Wu, L. Covalent Triazine-Based Frameworks as Visible Light Photocatalysts for the Splitting of Water. Macromol. Rapid Commun. 2015, 36 (20), 1799-1805.

(34) Kong, D.; Han, X.; Xie, J.; Ruan, Q.; Windle, C. D.; Gadipelli, S.; Shen, K.; Bai, Z.; Guo, Z.; Tang, J. Tunable Covalent TriazineBased Frameworks (CTF-0) for Visible-Light-Driven Hydrogen and Oxygen Generation from Water Splitting. ACS Catal. 2019, 9 (9), 7697-7707.

(35) Struijk, C. B.; Beerthuis, R. K.; Pabon, H. J. J.; van Dorp, D. A. Specificity in the enzymic conversion of polyunsaturated fatty acids into prostaglandins. Recueil des Travaux Chimiques des Pays-Bas 1966, 85 (12), 1233-1250.

(36) Gattrell, M.; Kirk, D. A Fourier Transform Infrared Spectroscopy Study of the Passive Film Produced during Aqueous Acidic Phenol Electro-oxidation. J. Electrochem. Soc. 1992, 139 (10), 2736-2744.

(37) Manecke, V. G.; Wöhrle, D. Synthese und halbleitereigenschaften einiger komplexe und der aus ihnen hergestellten polymeren. Teil 2. Polymere mit phthalocyaninartiger und triazinartiger stuktur. Makromol. Chem. 1968, 120 (1), 176-191.

(38) Liebl, M. R.; Senker, J. Microporous Functionalized TriazineBased Polyimides with High $\mathrm{CO}_{2}$ Capture Capacity. Chem. Mater. 2013, 25 (6), 970-980.

(39) Wu, W.-C.; Liao, L.-F.; Lien, C.-F.; Lin, J.-L. FTIR study of adsorption, thermal reactions and photochemistry of benzene on powdered $\mathrm{TiO}_{2}$. Phys. Chem. Chem. Phys. 2001, 3 (19), 4456-4461.

(40) Liu, B. H.; Dou, L. T.; He, F.; Yang, J.; Li, Z. P. A cobalt coordination compound with indole acetic acid for fabrication of a high performance cathode catalyst in fuel cells. RSC $A d v$. 2016, 6 (23), 19025-19033.

(41) Taha, M. A; Singh, S. R; Dennis, V. A Biodegradable PLGA85/ 15 nanoparticles as a delivery vehicle for Chlamydia trachomatis recombinant MOMP-187 peptide. Nanotechnology 2012, 23 (32), 325101.

(42) Larkin, P. Infrared and Raman Spectroscopy: Principles and Spectral Interpretation; Elsevier: 2017.

(43) Nakamoto, K.; Nakamoto, K. Infrared and Raman Spectra of Inorganic and Coordination Compounds; Wiley: 1977.

(44) Vyas, V. S.; Haase, F.; Stegbauer, L.; Savasci, G.; Podjaski, F.; Ochsenfeld, C.; Lotsch, B. V. A tunable azine covalent organic framework platform for visible light-induced hydrogen generation. Nat. Commun. 2015, 6, 8508.

(45) Hug, S.; Mesch, M. B.; Oh, H.; Popp, N.; Hirscher, M.; Senker, J.; Lotsch, B. V. A fluorene based covalent triazine framework with high $\mathrm{CO}_{2}$ and $\mathrm{H}_{2}$ capture and storage capacities. J. Mater. Chem. A 2014, 2 (16), 5928-5936.

(46) Thomas, A.; Fischer, A.; Goettmann, F.; Antonietti, M.; Muller, J.-O.; Schlogl, R.; Carlsson, J. M. Graphitic carbon nitride materials: variation of structure and morphology and their use as metal-free catalysts. J. Mater. Chem. 2008, 18 (41), 4893-4908.

(47) Muller, D. A.; Sorsch, T.; Moccio, S.; Baumann, F.; EvansLutterodt, K.; Timp, G. The electronic structure at the atomic scale of ultrathin gate oxides. Nature 1999, 399 (6738), 758-761. 
(48) Robin, M. B.; Ishii, I.; McLaren, R.; Hitchcock, A. P. Fluorination effects on the inner-shell spectra of unsaturated molecules. J. Electron Spectrosc. Relat. Phenom. 1988, 47, 53-92.

(49) Li, J.; Shen, B.; Hong, Z.; Lin, B.; Gao, B.; Chen, Y. A facile approach to synthesize novel oxygen-doped $\mathrm{g}-\mathrm{C}_{3} \mathrm{~N}_{4}$ with superior visible-light photoreactivity. Chem. Commun. 2012, 48 (98), 1201712019.

(50) Jorge, A. B.; Martin, D. J.; Dhanoa, M. T. S.; Rahman, A. S.; Makwana, N.; Tang, J.; Sella, A.; Corà, F.; Firth, S.; Darr, J. A.; McMillan, P. F. $\mathrm{H}_{2}$ and $\mathrm{O}_{2}$ Evolution from Water Half-Splitting Reactions by Graphitic Carbon Nitride Materials. J. Phys. Chem. C 2013, 117 (14), 7178-7185.

(51) Deifallah, M.; McMillan, P. F.; Corà, F. Electronic and Structural Properties of Two-Dimensional Carbon Nitride Graphenes. J. Phys. Chem. C 2008, 112 (14), 5447-5453.

(52) Martin, D. J.; Reardon, P. J. T.; Moniz, S. J. A.; Tang, J. Visible Light-Driven Pure Water Splitting by a Nature-Inspired Organic Semiconductor-Based System. J. Am. Chem. Soc. 2014, 136 (36), $12568-12571$

(53) Kudo, A.; Omori, K.; Kato, H. A Novel Aqueous Process for Preparation of Crystal Form-Controlled and Highly Crystalline $\mathrm{BiVO}_{4}$ Powder from Layered Vanadates at Room Temperature and Its Photocatalytic and Photophysical Properties. J. Am. Chem. Soc. 1999, 121 (49), 11459-11467.

(54) Xie, J.; Shevlin, S. A.; Ruan, Q.; Moniz, S. J. A.; Liu, Y.; Liu, X.; Li, Y.; Lau, C. C.; Guo, Z. X.; Tang, J. Efficient visible light-driven water oxidation and proton reduction by an ordered covalent triazinebased framework. Energy Environ. Sci. 2018, 11 (6), 1617-1624. 